\title{
NULL CONTROLLABILITY OF NONLINEAR CONVECTIVE HEAT EQUATIONS*
}

\author{
Sebastian Aniţa ${ }^{1}$ and Viorel Barbu ${ }^{1}$
}

\begin{abstract}
The internal and boundary exact null controllability of nonlinear convective heat equations with homogeneous Dirichlet boundary conditions are studied. The methods we use combine Kakutani fixed point theorem, Carleman estimates for the backward adjoint linearized system, interpolation inequalities and some estimates in the theory of parabolic boundary value problems in $L^{k}$.
\end{abstract}

AMS Subject Classification. 93B05, 35K05, 46B70, 46E35.

Received January 30, 1999. Revised December 24, 1999.

\section{INTRODUCTION}

This paper concerns the internal controllability of the system

$$
\begin{cases}y_{t}-\Delta y+\operatorname{div}(b(y(x, t)))=m(x) u(x, t), & (x, t) \in Q \\ y(x, t)=0, & (x, t) \in \Sigma \\ y(x, 0)=y_{0}(x), & x \in \Omega\end{cases}
$$

$(T \in(0,+\infty))$, where $\Omega$ is a bounded domain of $\mathbb{R}^{n}, n \geq 1$, with a smooth boundary $\partial \Omega, Q=\Omega \times(0, T)$ and $\Sigma=\partial \Omega \times(0, T)$. Here $\omega \subset \Omega$ is a nonempty open subset and $m$ is the characteristic function of $\omega$. We denote by $\Delta, \nabla$ and div the Laplace, gradient, respectively divergence operators with respect to $x$. The function $b: \mathbb{R} \rightarrow \mathbb{R}^{n}$ is supposed to belong to $W_{\text {loc }}^{2, \infty}\left(\mathbb{R} ; \mathbb{R}^{n}\right)$.

Equation $(1.1)_{1}$ describes the heat propagation or the gas diffusion when the flux has the form

$$
q(x, t)=-\nabla y(x, t)+b(y(x, t)), \quad(x, t) \in Q .
$$

The main results of this paper amount to saying that system (1.1) is exactly null controllable under certain smoothness assumptions on the initial data $y_{0}$ and growth conditions on the nonlinearity. The boundary exact null controllability will also be discussed.

System (1.1) is said to be null controllable if for every $T>0$ and for all $y_{0}$ in a suitable space, there are $(y, u) \in H^{2,1}(Q) \times L^{2}(Q)$ which satisfy $(1.1)$ and such that $y(x, T)=0$ a.e. $x \in \Omega$.

Keywords and phrases: Null controllability, Carleman estimates, interpolation inequality, Kakutani fixed point theorem.

* This work was supported by the grant CNSU/CNFIS 120(1997) of the World Bank and Romanian Government.

1 Faculty of Mathematics, University "Al.I. Cuza", 6600 Iaşi, Romania. 
We recall that the internal null controllability of the linear heat equation, when the control acts on a subset of the domain, was established by Lebeau and Robbiano [14] and was later extended to the semilinear equation

$$
y_{t}(x, t)-\Delta y(x, t)+f(y(x, t))=m(x) u(x, t), \quad(x, t) \in Q
$$

by Fursikov and Imanuvilov [11] in the sublinear case and by Barbu [3] and Fernández-Cara [8] in the superlinear case. A related result has been proved by Imanuvilov and Yamamoto [12] for parabolic equations in Sobolev spaces of negative order.

Approximate and local controllability results for superlinear heat equation of the form (1.2) were established in $[7,9]$ and $[17]$. An approximate controllability result for the heat equation

$$
y_{t}(x, t)-\Delta y(x, t)+f(y(x, t), \nabla y(x, t))=m(x) u(x, t), \quad(x, t) \in Q
$$

has been obtained by Zuazua [18] in the case when $f: \mathbb{R} \times \mathbb{R}^{n} \longrightarrow \mathbb{R}$ is globally Lipschitz.

The paper is organized as follows: the main results are stated in Section 2 and proved in the next sections via Kakutani fixed point theorem. The proofs are based on Carleman inequality for the backward adjoint linearized system associated with (1.1) and on some interpolation inequalities.

We shall use the standard notions for the Sobolev spaces $H^{k}(\Omega), H_{0}^{1}(\Omega)$ and the $L^{k}$ spaces on $\Omega$ and $Q$, $1 \leq k \leq+\infty$, with the norm denoted $\|\cdot\|_{k}$. Denote by $|\cdot|$ the usual norm of $\mathbb{R}^{n}$ and by $(\cdot, \cdot)$ the inner product of $L^{2}(\Omega)$.

Moreover, we set

$$
\begin{aligned}
& W_{k}^{2}(\Omega)=\left\{y \in L^{k}(\Omega) ; D_{x_{i}}^{s} y \in L^{k}(\Omega), s=1,2, i=1,2, \ldots, n\right\} \\
& W_{k}^{2,1}(Q)=\left\{y \in L^{k}(Q) ; D_{t}^{r} D_{x_{i}}^{s} y \in L^{k}(Q), 2 r+s \leq 2, i=1,2, \ldots, n\right\} \\
& 2 \leq k \leq+\infty \\
& W^{1,2}\left(0, T ; L^{2}(\Omega)\right)=\left\{y \in L^{2}\left(0, T ; L^{2}(\Omega)\right) ; \frac{d y}{d t} \in L^{2}\left(0, T ; L^{2}(\Omega)\right)\right\} \\
& H^{2,1}(Q)=W_{2}^{2,1}(Q) \cap L^{2}\left(0, T ; H_{0}^{1}(\Omega)\right),
\end{aligned}
$$

where $\frac{d y}{d t}$ and $D_{t}^{r} D_{x_{i}}^{s} y$ are taken in the sense of distributions.

For the definitions and basic properties of the fractional order spaces $W_{k}^{s}(\Omega)$ and $H^{s}(\Omega), s \geq 0, k>1$, we refer to [1].

\section{The MAIN RESUlts}

We set

$$
a=b^{\prime} \text { and } a_{0}=b^{\prime \prime} .
$$

Now we are ready to formulate the main result of this paper.

Theorem 2.1. Let $n=1$ and

$$
\begin{cases}|a(r)| \leq\left(1+\ln ^{\frac{1}{2}}(|r|+1)\right) \varphi(r), & \forall r \in \mathbb{R} \\ \left|a_{0}(r)\right| \leq\left(1+\ln ^{\frac{1}{2}}(|r|+1)\right)(|r|+1)^{-1} \varphi_{0}(r), & \text { a.e. } r \in \mathbb{R}\end{cases}
$$


where $\varphi, \varphi_{0}: \mathbb{R} \rightarrow \mathbb{R}$ are continuous functions such that $\lim _{|r| \rightarrow \infty} \varphi(r)=\lim _{|r| \rightarrow \infty} \varphi_{0}(r)=0$. Then the system (1.1) is exactly null controllable for all $y_{0} \in H_{0}^{1}(\Omega)$, i.e., for every $T \in(0,+\infty)$ there are $(y, u) \in H^{2,1}(Q) \times L^{2}(Q)$ which satisfy $(1.1)$ and $y(x, T)=0$ a.e. $x \in \Omega$.

Let $n=2,3$ and

$$
\begin{cases}|a(r)| \leq\left(1+\ln ^{\frac{1}{2}}(|r|+1)\right) \varphi(r), & \forall r \in \mathbb{R} \\ \left|a_{0}(r)\right| \leq\left(1+\ln ^{\frac{1-2 \delta}{2}}(|r|+1)\right)(|r|+1)^{-1} \varphi_{0}(r), & \text { a.e. } r \in \mathbb{R},\end{cases}
$$

where $\delta \in\left(0, \frac{1}{2}\right)$ and $\varphi, \varphi_{0}: \mathbb{R} \rightarrow \mathbb{R}$ are as above. Then the system (1.1) is exactly null controllable for all $y_{0} \in H_{0}^{1}(\Omega) \cap H^{2}(\Omega)$.

Remark 2.2. If $n=2$, the conclusion of the theorem remains true for all $y_{0} \in H_{0}^{1}(\Omega) \cap W_{k}^{2-\frac{2}{k}}(\Omega)$, where $k>2$ is arbitrary but fixed.

This is a more general case because there exists $k>2$ such that

$$
H^{2}(\Omega) \subset W_{k}^{2-\frac{2}{k}}(\Omega)
$$

algebraically and topologically (as a consequence of Th. 7.58 in [1]).

As expected, this result may be extended to a larger class of initial data by means of the smoothing effect of the heat equation. As regards the condition $1 \leq n \leq 3$, this is a restriction imposed by the Sobolev imbedding theorem (see Rem. 4.3 below).

Theorem 2.3. Under assumptions of Theorem 2.1, for each $y_{0} \in L^{2}(\Omega)$ and every $T \in(0,+\infty)$ there are $u \in L^{2}(Q)$,

$$
y \in C\left([0, T] ; L^{2}(\Omega)\right) \cap L^{2}\left(0, T ; H_{0}^{1}(\Omega)\right) \cap L^{2}\left(\varepsilon, T ; H^{2}(\Omega)\right)
$$

(for any $0<\varepsilon<T), a(y) \cdot \nabla y \in L^{1}(Q)$, which satisfy (1.1) and such that $y(x, T)=0$ a.e. $x \in \Omega$.

In the latter case the solution $y$ to (1.1) is considered in the weak or mild sense, i.e.,

$$
y(t)=S(t) y_{0}-\int_{0}^{t} S(t-s)(a(y(s)) \cdot \nabla y(s)) d s+\int_{0}^{t} S(t-s)(m u(s)) d s
$$

$t \in[0, T]$, where $S(t), t \geq 0$ is the semigroup generated on $L^{1}(\Omega)$ by $A=\Delta$,

$$
D(A)=\left\{y \in W_{0}^{1,1}(\Omega) ; \Delta y \in L^{1}(\Omega)\right\}
$$

(see e.g. $[2,5])$.

In the same manner as in [3] one can prove the exact boundary null controllability result as a consequence of Theorem 2.1. 
Corollary 2.4. Under the assumptions in Theorem 2.1, there are $v \in L^{2}\left(\Sigma_{0}\right)$ and $y \in W_{2}^{2,1}(Q)$ such that

$$
\left\{\begin{array}{l}
y_{t}-\Delta y+\operatorname{div}(b(y))=0, \\
y= \begin{cases}v, \quad(x, t) \in \Sigma_{0} & (x, t) \in Q \\
0, \quad(x, t) \in \Sigma \backslash \Sigma_{0} & \\
y(x, 0)=y_{0}(x), & x \in \Omega \\
y(x, T)=0, & x \in\end{cases}
\end{array}\right.
$$

Here $\Sigma_{0}=\Sigma \cap B\left(x_{0} ; \varepsilon\right)$, where $x_{0} \in \partial \Omega$ and $\varepsilon>0$ is a constant (which may be choosen as small as we wish).

If we denote by

$$
\rho(r)=\sup \{|a(w)| ; w \in \mathbb{R},|w| \leq r\}
$$

and

$$
\rho_{0}(r)=\operatorname{ess} \sup \left\{\left|a_{0}(w)\right| ; w \in \mathbb{R},|w| \leq r\right\}
$$

we immediately remark that (2.1) implies that

$$
\lim _{r \rightarrow \infty}\left(r e^{-\mu\left(\rho^{2}(r)+\rho_{0}^{2}(r) r^{2}\right)}\right)=+\infty, \quad \forall \mu>0
$$

and that (2.2) implies that

$$
\lim _{r \rightarrow \infty}\left(r e^{-\mu\left(\rho^{2}(r)+\left(\rho_{0}(r) r\right)^{\frac{2}{1-2 \delta}}\right)}\right)=+\infty, \quad \forall \mu>0 .
$$

Conditions $(2.1)^{\prime}$ and $(2.2)^{\prime}$ will be used in the next two sections.

Remark 2.5. Consider now the exact null controllability problem of the following more general equation

$$
\begin{cases}y_{t}-\Delta y+\operatorname{div}(b(y(x, t)))+f(x, t, y(x, t))=m(x) u(x, t), & (x, t) \in Q \\ y(x, t)=0, & (x, t) \in \Sigma \\ y(x, 0)=y_{0}(x), & x \in \Omega\end{cases}
$$

Suppose that the hypotheses in Theorem 2.1 hold and the function $f: Q \times \mathbb{R} \rightarrow \mathbb{R}$ is continuous in the third variable, measurable in $(x, t)$ and satisfies

$$
\begin{gathered}
f(x, t, r) r \geq-\mu_{0} r^{2}, \quad \forall r \in \mathbb{R}, \quad(x, t) \in Q, \\
|f(x, t, r)| \leq|r| \xi(r)\left(1+(\ln (1+|r|))^{\frac{3}{2}}\right), \quad \forall(x, t, r) \in Q \times \mathbb{R},
\end{gathered}
$$

where $\mu_{0} \geq 0$ is a constant and $\xi: \mathbb{R} \rightarrow \mathbb{R}$ is a continuous function, such that $\lim _{|r| \rightarrow \infty} \xi(r)=0$ ( $f$ satisfies the assumptions in [3]).

Combining the estimates in our paper and those in [3] the exact null controllability of (2.5) can be proved for all $y_{0} \in H_{0}^{1}(\Omega) \cap H^{2}(\Omega)$, if $n=1$ and $y_{0} \in H_{0}^{1}(\Omega) \cap W_{\frac{5}{2}}^{2}(\Omega)$, if $n \in\{2,3)$.

The controllability result of [3] was recently extended in [10] to functions $f$ which do not satisfy the growth condition (2.6). One might suspect that the later result remains true for system (2.5) as well. 


\section{Proof of Theorem 2.1 For $n=1,2$}

We fix

where $k>2$ is arbitrary but fixed and define

$$
\begin{array}{ll}
y_{0} \in H_{0}^{1}(\Omega), & \text { if } n=1, \\
y_{0} \in H_{0}^{1}(\Omega) \cap W_{k}^{2-\frac{2}{k}}(\Omega), & \text { if } n=2,
\end{array}
$$

$$
K=\left\{w \in L^{\infty}(Q) ;\|w(t)\|_{\infty} \leq M,\|\nabla w(t)\|_{2} \leq M \text { a.e. } t \in(0, T)\right\}
$$

where $M$ is an arbitrary but fixed positive constant.

For any arbitrary, but fixed $w \in K$ consider the exact null controllability problem for

$$
\begin{cases}y_{t}-\Delta y+a(w) \cdot \nabla y=m(x) u(x, t), & (x, t) \in Q \\ y=0, & (x, t) \in \Sigma \\ y(x, 0)=y_{0}(x), & x \in \Omega,\end{cases}
$$

where $m$ is the characteristic function of $\omega$ and $u \in L^{2}(Q)$.

In what follows we shall use the general Carleman inequality for linear parabolic equations given in [11]. Namely, let $\tilde{\omega} \subset \subset \omega$ be a nonempty bounded set and $\psi \in C^{2}(\bar{\Omega})$ be such that

$$
\begin{aligned}
& \psi(x)>0, \forall x \in \Omega, \quad \psi(x)=0, \forall x \in \partial \Omega \\
& |\nabla \psi(x)|>0, \forall x \in \bar{\Omega} \backslash \widetilde{\omega}
\end{aligned}
$$

and set

$$
\alpha(x, t)=\frac{e^{\lambda \psi(x)}-e^{2 \lambda\|\psi\|_{C(\bar{\Omega})}}}{t(T-t)},
$$

where $\lambda$ is an appropriate positive constant.

The following result holds:

Lemma 3.1. There exist positive constants $C_{1}, s_{1}$ such that

$$
\left\{\begin{array}{l}
\frac{1}{s} \int_{Q} t(T-t) e^{2 s \alpha}\left(\left|q_{t}\right|^{2}+|\Delta q|^{2}\right) d x d t+s \int_{Q} \frac{e^{2 s \alpha}}{t(T-t)}|\nabla q|^{2} d x d t \\
+s^{3} \int_{Q} \frac{e^{2 s \alpha}}{t^{3}(T-t)^{3}}|q|^{2} d x d t \\
\leq C_{1}\left[\int_{Q} e^{2 s \alpha}\left|q_{t}+\Delta q\right|^{2} d x d t+s^{3} \int_{\omega \times(0, T)} \frac{e^{2 s \alpha}}{t^{3}(T-t)^{3}}|q|^{2} d x d t\right]
\end{array}\right.
$$

for all $q \in C^{2}(\bar{Q}), q=0$ on $\Sigma$ and $s \geq s_{1}$.

One knows that for each $y_{0} \in L^{2}(\Omega)$ and $w \in K$, the exact null controllability problem associated to (3.2) has at least one solution $(u, y)$.

In Lemma 3.2 below we shall prove some estimates on such a solution.

In the following we shall denote by the same symbol $C$, several constants independent of $y_{0}, w, M$ and all other variables. 
Lemma 3.2. Let $k>2$. For each $w \in K$ and

$$
\begin{array}{ll}
y_{0} \in H_{0}^{1}(\Omega), & \text { for } n=1 \\
y_{0} \in H_{0}^{1}(\Omega) \cap W_{k}^{2-\frac{2}{k}}(\Omega), & \text { for } n=2,
\end{array}
$$

there are $y \in L^{2}\left(0, T ; H_{0}^{1}(\Omega)\right) \cap W_{k}^{2,1}(Q)$ and $u \in L^{k}(Q)$, which satisfy $(3.2)$,

$$
\begin{aligned}
& y(x, T)=0, \quad \text { a.e. } x \in \Omega \\
& \|m u\|_{L^{l}(Q)}^{2} \leq C_{l} A(M)\left\|y_{0}\right\|_{2}^{2},
\end{aligned}
$$

for any $l \in(2,+\infty)$, where $C_{l}$ is a positive constant independent of $y_{0}, w$ and $M$ and

$$
A(M)= \begin{cases}e^{C\left[\rho^{2}(M)+\rho_{0}^{2}(M) M^{2}\right]}, & \text { if } n=1 \\ e^{C\left[\rho^{2}(M)+\left(\rho_{0}(M) M\right)^{\left.\frac{2}{1-2 \delta}\right]},\right.} & \text { if } n=2,\end{cases}
$$

$\left(\rho(M)\right.$ and $\rho_{0}(M)$ are defined by (2.3) and (2.4), respectively).

Proof. For any $\varepsilon>0$ consider the optimal control problem

$$
\text { Minimize } \int_{Q} e^{-2 s \alpha} t^{3}(T-t)^{3} u^{2} d x d t+\frac{1}{\varepsilon} \int_{\Omega} y^{2}(x, T) d x
$$

subject to $(3.2)$.

By standard arguments it follows there is an optimal pair $\left(u_{\varepsilon}, y_{\varepsilon}\right)$ and by the maximum principle (see e.g. [4]) we have

$$
u_{\varepsilon}(x, t)=m(x) p_{\varepsilon}(x, t) e^{2 s \alpha} t^{-3}(T-t)^{-3}, \quad \text { a.e. }(x, t) \in Q,
$$

where $p_{\varepsilon} \in H^{2,1}(Q)$ is the solution of

$$
\begin{cases}p_{t}+\Delta p+\operatorname{div}(a(w) p)=0, & (x, t) \in Q \\ p=0, & (x, t) \in \Sigma \\ p(x, T)=-\frac{1}{\varepsilon} y_{\varepsilon}(x, T), & x \in \Omega .\end{cases}
$$

Multiplying (3.5) by $y_{\varepsilon}$ and integrating on $Q$, we get after some calculation (and using (3.4)) that

$$
\begin{aligned}
\int_{\omega \times(0, T)} e^{-2 s \alpha} t^{3}(T-t)^{3} u_{\varepsilon}^{2}(x, t) d x d t+\frac{1}{\varepsilon} \int_{\Omega} y_{\varepsilon}^{2}(x, T) d x & =\int_{\omega \times(0, T)} e^{2 s \alpha} t^{-3}(T-t)^{-3} p_{\varepsilon}^{2}(x, t) d x d t+\frac{1}{\varepsilon} \int_{\Omega} y_{\varepsilon}^{2}(x, T) d x \\
& =-\int_{\Omega} y_{0}(x) p_{\varepsilon}(x, 0) d x
\end{aligned}
$$

It is obvious that (3.5) can be equivalently written as

$$
\begin{cases}p_{t}+\Delta p+a(w) \cdot \nabla p+\left(a_{0}(w) \nabla w\right) p=0, & (x, t) \in Q \\ p=0, & (x, t) \in \Sigma \\ p(x, T)=-\frac{1}{\varepsilon} y_{\varepsilon}(x, T), & x \in \Omega\end{cases}
$$


and by Carleman's inequality (3.3) we infer that

$$
\begin{aligned}
& \int_{Q} e^{2 s \alpha}\left[\frac{t(T-t)}{s}\left(\left|\left(p_{\varepsilon}\right)_{t}\right|^{2}+\left|\Delta p_{\varepsilon}\right|^{2}\right)+\frac{s}{t(T-t)}\left|\nabla p_{\varepsilon}\right|^{2}+\frac{s^{3}}{t^{3}(T-t)^{3}}\left|p_{\varepsilon}\right|^{2}\right] d x d t \\
& \leq C_{1} \int_{Q} e^{2 s \alpha}\left(|a(w)|^{2}\left|\nabla p_{\varepsilon}\right|^{2}+\left|a_{0}(w)\right|^{2}|\nabla w|^{2}\left|p_{\varepsilon}\right|^{2}\right) d x d t \\
& +C_{1} s^{3} \int_{\omega \times(0, T)} e^{2 s \alpha} t^{-3}(T-t)^{-3}\left|p_{\varepsilon}\right|^{2} d x d t .
\end{aligned}
$$

Denote by

$$
\begin{aligned}
& I_{1}(w)=\int_{Q} e^{2 s \alpha}|a(w)|^{2}\left|\nabla p_{\varepsilon}\right|^{2} d x d t \\
& I_{2}(w)=\int_{Q} e^{2 s \alpha}\left|a_{0}(w)\right|^{2}|\nabla w|^{2}\left|p_{\varepsilon}\right|^{2} d x d t .
\end{aligned}
$$

By (2.3) and (2.4) we conclude that

$$
I_{1}(w) \leq \rho^{2}(M) \int_{Q} e^{2 s \alpha}\left|\nabla p_{\varepsilon}\right|^{2} d x d t
$$

and

$$
I_{2}(w) \leq \rho_{0}^{2}(M) \int_{Q} e^{2 s \alpha}|\nabla w|^{2}\left|p_{\varepsilon}\right|^{2} d x d t .
$$

Recalling that $H^{2}(\Omega) \subset C(\bar{\Omega})$ for $n \leq 2$ and using the Sobolev imbedding theorem we get

$$
\begin{gathered}
\int_{Q} e^{2 s \alpha}|\nabla w|^{2}\left|p_{\varepsilon}\right|^{2} d x d t=\int_{0}^{T} \int_{\Omega}|\nabla w|^{2}\left|p_{\varepsilon} e^{s \alpha}\right|^{2} d x d t \leq \int_{0}^{T}\left\|p_{\varepsilon}(t) e^{s \alpha(t)}\right\|_{\infty}^{2} \int_{\Omega}|\nabla w(t)|^{2} d x d t \\
\leq\|\nabla w\|_{L^{\infty}\left(0, T ; L^{2}(\Omega)\right)}^{2} \int_{0}^{T}\left\|p_{\varepsilon}(t) e^{s \alpha(t)}\right\|_{\infty}^{2} d t
\end{gathered}
$$

and as a consequence we have

$$
I_{2}(w) \leq C \rho_{0}^{2}(M) M^{2} \int_{0}^{T}\left\|p_{\varepsilon}(t) e^{s \alpha(t)}\right\|_{\infty}^{2} d t \leq \begin{cases}C \rho_{0}^{2}(M) M^{2} \int_{0}^{T}\left\|p_{\varepsilon}(t) e^{s \alpha(t)}\right\|_{H_{0}^{1}(\Omega)}^{2} d t, & \text { if } n=1 \\ C \rho_{0}^{2}(M) M^{2} \int_{0}^{T}\left\|p_{\varepsilon}(t) e^{s \alpha(t)}\right\|_{H^{1+\delta}(\Omega)}^{2} d t, & \text { if } n=2 .\end{cases}
$$

We have used the inclusions

$$
\begin{array}{ll}
H_{0}^{1}(\Omega) \subset L^{\infty}(\Omega), & \text { if } n=1, \\
H^{1+\zeta}(\Omega) \subset L^{\infty}(\Omega), & \text { if } n=2
\end{array}
$$

for any $\zeta>0$ (algebraically and topologically).

If $n=1$, we have that

$$
C_{1} I_{2}(w) \leq C \rho_{0}^{2}(M) M^{2}\left(\int_{Q} e^{2 s \alpha}\left|\nabla p_{\varepsilon}\right|^{2} d x d t+s^{2} \int_{Q} \frac{e^{2 s \alpha}}{t^{2}(T-t)^{2}}\left|p_{\varepsilon}\right|^{2} d x d t\right)
$$


and using (3.7-3.10) we obtain that

$$
\begin{aligned}
& \int_{Q}\left[\frac{s^{3}}{t^{3}(T-t)^{3}}\left|p_{\varepsilon}\right|^{2}+\frac{s}{t(T-t)}\left|\nabla p_{\varepsilon}\right|^{2}+\frac{t(T-t)}{s}\left(\left|\left(p_{\varepsilon}\right)_{t}\right|^{2}+\left|\Delta p_{\varepsilon}\right|^{2}\right)\right] e^{2 s \alpha} d x d t \\
& \leq C \int_{\omega \times(0, T)} \frac{s^{3}}{t^{3}(T-t)^{3}}\left|p_{\varepsilon}\right|^{2} e^{2 s \alpha} d x d t,
\end{aligned}
$$

for any $s \geq C\left[\rho^{2}(M)+\rho_{0}^{2}(M) M^{2}\right]$.

If $n=2$, we shall use the interpolation inequality

$$
\|z\|_{H^{1+\delta}(\Omega)} \leq C\|z\|_{H^{2}(\Omega)}^{\delta} \cdot\|z\|_{H^{1}(\Omega)}^{1-\delta},
$$

$\forall z \in H^{2}(\Omega)$ (see $\left.[15,16]\right)$. This implies that

$$
\|z\|_{H^{1+\delta}(\Omega)}^{2} \leq C\|\Delta z\|_{2}^{2 \delta} \cdot\|\nabla z\|_{2}^{2(1-\delta)} \leq \frac{t(T-t)}{s}\|\Delta z\|_{2}^{2}+C\left[\frac{s}{t(T-t)}\right]^{\frac{\delta}{1-\delta}} \cdot\|\nabla z\|_{2}^{2},
$$

$\forall z \in H^{2}(\Omega) \cap H_{0}^{1}(\Omega), \forall t \in(0, T), \forall s>0$. From (3.10) and (3.12) we obtain that

$$
\begin{aligned}
C_{1} I_{2}(w) & \leq \int_{Q} \frac{t(T-t)}{s}\left|\Delta\left(p_{\varepsilon} e^{s \alpha}\right)\right|^{2} d x d t+C \rho_{0}^{2}(M) M^{2} \int_{Q}\left[\frac{s \rho_{0}(M)^{2} M^{2}}{t(T-t)}\right]^{\frac{\delta}{1-\delta}} \cdot\left|\nabla\left(p_{\varepsilon} e^{s \alpha}\right)\right|^{2} d x d t \\
& \leq \int_{Q} \frac{t(T-t)}{s}\left|\Delta\left(p_{\varepsilon} e^{s \alpha}\right)\right|^{2} d x d t+C\left[\rho_{0}(M) M\right]^{\frac{2}{1-\delta}} \cdot s^{\frac{2 \delta-1}{1-\delta}} \int_{Q} \frac{s}{t(T-t)}\left|\nabla\left(p_{\varepsilon} e^{s \alpha}\right)\right|^{2} d x d t .
\end{aligned}
$$

Taking now

we obtain via (3.7-3.9) and (3.13) that

$$
s \geq C\left[\rho^{2}(M)+\left(\rho_{0}(M) M\right)^{\frac{2}{1-2 \delta}}\right],
$$

$$
\begin{aligned}
\int_{Q}\left[\frac{s^{3}}{t^{3}(T-t)^{3}}\left|p_{\varepsilon}\right|^{2}\right. & \left.+\frac{s}{t(T-t)}\left|\nabla p_{\varepsilon}\right|^{2}+\frac{t(T-t)}{s}\left(\left|\left(p_{\varepsilon}\right)_{t}\right|^{2}+\left|\Delta p_{\varepsilon}\right|^{2}\right)\right] e^{2 s \alpha} d x d t \\
& \leq C \int_{\omega \times(0, T)} \frac{s^{3}}{t^{3}(T-t)^{3}}\left|p_{\varepsilon}\right|^{2} e^{2 s \alpha} d x d t
\end{aligned}
$$

for any $s \geq C\left[\rho^{2}(M)+\left(\rho_{0}(M) M\right)^{\frac{2}{1-2 \delta}}\right]$.

Multiplying now (3.6), by $p_{\varepsilon}$ and integrating over $\Omega$ we get

$$
\begin{aligned}
\frac{1}{2} \cdot \frac{d}{d t} \int_{\Omega}\left|p_{\varepsilon}(x, t)\right|^{2} d x-\int_{\Omega}\left|\nabla p_{\varepsilon}(t)\right|^{2} d x & =-\int_{\Omega}\left(a(w) \cdot \nabla p_{\varepsilon}\right) p_{\varepsilon} d x-\int_{\Omega}\left(a_{0}(w) \nabla w\right)\left|p_{\varepsilon}\right|^{2} d x \\
& \geq-\int_{\Omega}\left|\nabla p_{\varepsilon}(t)\right|^{2} d x-C\left[\rho^{2}(M)+\rho_{0}^{2}(M) M^{2}\right] \int_{\Omega}\left|p_{\varepsilon}(t)\right|^{2} d x
\end{aligned}
$$

and consequently

$$
\int_{\Omega}\left|p_{\varepsilon}(x, 0)\right|^{2} d x \leq C e^{C\left[\rho^{2}(M)+\rho_{0}^{2}(M) M^{2}\right]} \int_{Q}\left|p_{\varepsilon}\right|^{2} \frac{1}{t^{3}(T-t)^{3}} e^{2 s \alpha} d x d t
$$


for any $s \geq s_{0}+C\left[\rho^{2}(M)+\rho_{0}^{2}(M) M^{2}\right]$.

Relations $(3.11,3.14)$ and (3.15) imply that

$$
\int_{\Omega}\left|p_{\varepsilon}(x, 0)\right|^{2} d x \leq A(M) \int_{\omega \times(0, T)}\left|p_{\varepsilon}(x, t)\right|^{2} \frac{e^{2 s \alpha}}{t^{3}(T-t)^{3}} d x d t
$$

for $s \geq s_{0}+A(M)$.

By (3.16) we get after some calculation that

$$
\begin{aligned}
& \int_{\omega \times(0, T)} e^{-2 s \alpha} t^{3}(T-t)^{3}\left|u_{\varepsilon}(x, t)\right|^{2} d x d t+\frac{1}{\varepsilon} \int_{\Omega}\left|y_{\varepsilon}(x, T)\right|^{2} d x \\
& =\int_{\omega \times(0, T)} e^{2 s \alpha} t^{-3}(T-t)^{-3}\left|p_{\varepsilon}(x, t)\right|^{2} d x d t+\frac{1}{\varepsilon} \int_{\Omega}\left|y_{\varepsilon}(x, T)\right|^{2} d x \\
& \leq\left\{\begin{array}{c}
C\left\|y_{0}\right\|_{2}^{2} e^{C\left[\rho^{2}(M)+\rho_{0}^{2}(M) M^{2}\right]}, \quad \text { if } n=1, \\
\text { for } s=s_{0}+C\left[\rho^{2}(M)+\rho_{0}^{2}(M) M^{2}\right] \\
C\left\|y_{0}\right\|_{2}^{2} e^{C\left[\rho^{2}(M)+\left(\rho_{0}(M) M\right)^{\frac{2}{1-2 \delta}}\right]}, \quad \text { if } n=2, \\
\text { for } s=s_{0}+C\left[\rho^{2}(M)+\left(\rho_{0}(M) M\right)^{\frac{2}{1-2 \delta}}\right] .
\end{array}\right.
\end{aligned}
$$

We set $v_{\varepsilon}=e^{2 s \alpha} t^{-3}(T-t)^{-3} p_{\varepsilon}$. Using $(3.11,3.14)$ and (3.17) we infer that

$$
\int_{Q} e^{2 s \alpha}\left[\frac{s^{3}}{t^{3}(T-t)^{3}}\left|p_{\varepsilon}\right|^{2}+\frac{s}{t(T-t)}\left|\nabla p_{\varepsilon}\right|^{2}+\frac{t(T-t)}{s}\left(\left|\left(p_{\varepsilon}\right)_{t}\right|^{2}+\left|\Delta p_{\varepsilon}\right|^{2}\right)\right] d x d t \leq A(M)\left\|y_{0}\right\|_{2}^{2}, \quad \forall \varepsilon>0 .
$$

This yields

$$
\left\|v_{\varepsilon}\right\|_{H^{2,1}(Q)}^{2} \leq A(M)\left\|y_{0}\right\|_{2}^{2} .
$$

Since $H^{2,1}(Q) \subset L^{l}(Q), \forall l \in(2,+\infty)$ (for $n=1,2$ ) we infer that

$$
\left\|m u_{\varepsilon}\right\|_{L^{l}(Q)}^{2} \leq C_{l} A(M)\left\|y_{0}\right\|_{2}^{2}
$$

for any $\varepsilon>0$, where $C_{l}$ depends on $l \in(2,+\infty)$.

This estimate and the existence theory of parabolic boundary value problems in $L^{l}(Q)$ (see [13]) imply that on a subsequence we have

$$
\begin{aligned}
& u_{\varepsilon} \quad \longrightarrow \quad u \quad \text { weakly in } L^{l}(Q) \\
& y_{\varepsilon} \longrightarrow y \quad \text { weakly in } L^{2}\left(0, T ; H_{0}^{1}(\Omega)\right) \cap W_{l}^{2,1}(Q) \text {, }
\end{aligned}
$$

where $(u, y)$ satisfy $(3.2)$ and $y(x, T)=0$ a.e. $x \in \Omega$. Moreover, (3.18) implies the estimate in Lemma 3.2 and this completes the proof of lemma.

Proof of Theorem 2.1 (continued). For each $w \in K$ denote by $\Phi(w) \subset L^{2}(Q)$ the set of all solutions $y^{u} \in$ $\cap_{l=2}^{\infty} W_{l}^{2,1}(Q) \cap L^{2}\left(0, T ; H_{0}^{1}(\Omega)\right)$ of $(3.2)$ such that $y^{u}(x, T)=0$, a.e. $x \in \Omega$ and

$$
\|m u\|_{L^{l}(Q)}^{2} \leq C_{l} A(M)\left\|y_{0}\right\|_{2}^{2}, \quad \forall l \in(2,+\infty)
$$


If we multiply (3.2) by $y_{t}^{u}-\Delta y^{u}$ and integrate on $\Omega \times(0, t)$, we obtain that

$$
\begin{aligned}
& \int_{0}^{t} \int_{\Omega}\left(\left|y_{t}^{u}\right|^{2}+\left|\Delta y^{u}\right|^{2}\right) d x d t+\frac{1}{2} \int_{\Omega}\left|\nabla y^{u}(t)\right|^{2} d x \\
& \leq \frac{1}{2} \int_{\Omega}\left|\nabla y_{0}\right|^{2} d x+2 \int_{0}^{t} \int_{\Omega}|a(w)|^{2}\left|\nabla y^{u}\right|^{2} d x d s+2 \int_{0}^{t} \int_{\Omega} m(x)|u(x, t)|^{2} d x d t
\end{aligned}
$$

and

$$
\int_{\Omega}\left|\nabla y^{u}(t)\right|^{2} d x \leq C e^{4 \rho^{2}(M) T}\left(\left\|y_{0}\right\|_{H_{0}^{1}(\Omega)}^{2}+\|m u\|_{L^{2}(Q)}^{2}\right) \leq A(M)\left\|y_{0}\right\|_{H_{0}^{1}(\Omega)}^{2} .
$$

It is readly seen that $\Phi(w)$ is a closed (this follows by (3.19) and (3.20)) and convex subset of $L^{2}(Q)$.

Moreover, Lemma 3.2 implies

$$
\left\|y^{u}\right\|_{L^{\infty}(Q)}^{2} \leq \begin{cases}A(M)\left\|y_{0}\right\|_{H_{0}^{1}(\Omega)}^{2}, & \text { if } n=1 \\ C\left\|y^{u}\right\|_{W_{k}^{2,1}(Q)}^{2} \leq A(M)\left\|y_{0}\right\|_{W_{k}^{2-\frac{2}{k}}(\Omega)}^{2}, & \text { if } n=2\end{cases}
$$

(see [13]).

Thus it follows by (3.20) and (3.21) that if

$$
\left\|y_{0}\right\|_{H_{0}^{1}(\Omega)} \leq C M e^{-C\left[\rho^{2}(M)+\rho_{0}^{2}(M) M^{2}\right]}, \text { for } n=1
$$

and

$$
\left\|y_{0}\right\|_{W_{k}^{2-\frac{2}{k}(\Omega)}} \leq C M e^{-C\left[\rho^{2}(M)+\left(\rho_{0}(M) M\right)^{\frac{2}{1-2 \delta}}\right]}, \text { for } n=2
$$

then we obtain via (3.1) that $\Phi(K) \subset K$.

Moreover, by estimate (3.19) it follows $\Phi(K)$ is a relatively compact subset of $L^{2}(Q)$.

Note also that $\Phi$ is upper semicontinuous in $L^{2}(Q) \times L^{2}(Q)$. Indeed, let $w_{n} \longrightarrow w$ in $L^{2}(Q), w_{n} \in K$ and $y_{n} \longrightarrow y$ in $L^{2}(Q), y_{n} \in \Phi\left(w_{n}\right), y_{n}=y^{u_{n}}$. By Lemma 3.2 it follows (selecting a subsequence if necessary) that

$$
\begin{array}{rll}
w_{n}(x, t) & \longrightarrow w(x, t) & \text { a.e. in } Q \\
u_{n} & \longrightarrow u & \text { weakly in } L^{2}(Q) \\
y_{n} & \longrightarrow y & \text { strongly in } C\left([0, T] ; L^{2}(\Omega)\right) \text { and } \\
& & \\
& & \text { weakly in } H^{2,1}(Q) .
\end{array}
$$

Since $y_{n}$ is a solution of

$$
\begin{cases}y_{t}-\Delta y+a\left(w_{n}(x, t)\right) \cdot \nabla y=m u_{n}(x, t), & (x, t) \in Q \\ y=0, & (x, t) \in \Sigma \\ y(x, 0)=y_{0}(x), y(x, T)=0, & x \in \Omega,\end{cases}
$$

we conclude (by passing to the limit) that $y \in \Phi(w)$ as claimed.

Then applying the Kakutani fixed point theorem (see [2], p. 7 and [6], p. 310) in the space $L^{2}(Q)$ to the mapping $\Phi$ we infer that there is at least one $w \in K$ such that $w \in \Phi(w)$. By definition of $\Phi$ this implies that there is at least one pair $(u, y)$ satisfying the conditions of Theorem 2.1.

Since $(2.1)^{\prime}$ and $(2.2)^{\prime}$ are satisfied we may infer that if

$$
\left\|y_{0}\right\|_{H_{0}^{1}(\Omega)}<C \sup \left\{r e^{-C\left[\rho^{2}(r)+\rho_{0}^{2}(r) r^{2}\right]} ; r>0\right\}=+\infty, \text { for } n=1
$$


and

$$
\left\|y_{0}\right\|_{W_{k}^{2-\frac{2}{k}}(\Omega)}<C \sup \left\{r e^{-C\left[\rho^{2}(r)+\left(\rho_{0}(r) r\right)^{\frac{2}{1-2 \delta}}\right]} ; r>0\right\}=+\infty, \text { for } n=2,
$$

then there is at least one pair $(u, y)$ satisfying the conditions of Theorem 2.1. This completes the proof for $n=1$ and $n=2$.

\section{Proof of Theorem 2.1 FOR $n=3$}

We fix $y_{0} \in H^{2}(\Omega) \cap H_{0}^{1}(\Omega)$ and define the set

$$
\begin{gathered}
K=\left\{w \in L^{\infty}(Q) \cap L^{\infty}\left(0, T ; H_{0}^{1}(\Omega) \cap H^{\frac{3}{2}-\delta}(\Omega)\right) ;\right. \\
\left.\|w(t)\|_{\infty} \leq M,\|w(t)\|_{H^{\frac{3}{2}-\delta}(\Omega)} \leq M \text { a.e. } t \in(0, T)\right\},
\end{gathered}
$$

where $M$ is an arbitrary but fixed positive constant.

For any arbitrary, but fixed $w \in K$ consider the exact null controllability problem for (3.2), where

$$
\begin{aligned}
& m \in C_{0}^{\infty}(\omega), \quad 0 \leq m(x) \leq 1, \forall x \in \omega \\
& m(x)=1, \forall x \in \omega_{0} .
\end{aligned}
$$

Here $\omega_{0}$ is a nonempty open subset of $\omega$ and $u \in L^{2}(Q)$.

By Lemma 3.2 we have the existence of two positive constants $C_{1}, s_{1}$, such that

$$
\begin{aligned}
& \frac{1}{s} \int_{Q} t(T-t) e^{2 s \alpha}\left(\left|q_{t}\right|^{2}+|\Delta q|^{2}\right) d x d t+s \int_{Q} \frac{e^{2 s \alpha}}{t(T-t)}|\nabla q|^{2} d x d t+s^{3} \int_{Q} \frac{e^{2 s \alpha}}{t^{3}(T-t)^{3}}|q|^{2} d x d t \\
& \leq C_{1}\left[\int_{Q} e^{2 s \alpha}\left|q_{t}+\Delta q\right|^{2} d x d t+s^{3} \int_{\omega_{0} \times(0, T)} \frac{e^{2 s \alpha}}{t^{3}(T-t)^{3}}|q|^{2} d x d t\right],
\end{aligned}
$$

for all $q \in C^{2}(\bar{Q}), q=0$ on $\Sigma$ and $s \geq s_{1}$.

Here $\alpha$ is defined in Section 3, for $\omega:=\omega_{0}$.

For each $y_{0} \in L^{2}(\Omega)$ and $w \in K$, the exact null controllability problem associated to (3.2) has at least one solution $(u, y)$.

Lemma 4.1 below is the main ingredient of the proof.

Lemma 4.1. For each $w \in K$ and $y_{0} \in H_{0}^{1}(\Omega) \cap H^{2}(\Omega)$, there are $y \in H^{2,1}(Q)$ and $u \in H^{2,1}(Q)$ which satisfy (3.2) and

$$
\begin{aligned}
& y(x, T)=0 \quad \text { a.e. } x \in \Omega \\
& \|m u\|_{H^{2,1}(Q)}^{2} \leq C\left\|y_{0}\right\|_{2}^{2} e^{C\left[\rho^{2}(M)+\left(\rho_{0}(M) M\right)^{\frac{2}{1-2 \delta}}\right]} .
\end{aligned}
$$

Proof. Consider the optimal control problem

$$
\text { Minimize } \int_{Q} e^{-2 s \alpha} t^{3}(T-t)^{3} m(x) u^{2}(x, t) d x d t+\frac{1}{\varepsilon} \int_{\Omega} y^{2}(x, T) d x
$$


subject to $u \in L^{2}(Q)$ and $y$ the solution of (3.2). There exists at least one optimal pair $\left(u_{\varepsilon}, y_{\varepsilon}\right)$ and by the maximum principle we have

$$
u_{\varepsilon}(x, t)=m(x) p_{\varepsilon}(x, t) e^{2 s \alpha} t^{-3}(T-t)^{-3} \quad \text { a.e. }(x, t) \in Q,
$$

where $p_{\varepsilon} \in H^{2,1}(Q)$ is the solution of (3.6).

In the same manner as in the previous section it follows

$$
\begin{aligned}
& \int_{Q} e^{2 s \alpha}\left[\frac{t(T-t)}{s}\left|\Delta p_{\varepsilon}\right|^{2}+\frac{s}{t(T-t)}\left|\nabla p_{\varepsilon}\right|^{2}+\frac{s^{3}}{t^{3}(T-t)^{3}}\left|p_{\varepsilon}\right|^{2}\right] d x d t \\
& \leq C_{1} \rho^{2}(M) \int_{Q} e^{2 s \alpha}\left|\nabla p_{\varepsilon}\right|^{2} d x d t+C_{1} \rho_{0}^{2}(M) \int_{Q} e^{2 s \alpha}|\nabla w|^{2}\left|p_{\varepsilon}\right|^{2} d x d t \\
& +C_{1} s^{3} \int_{\omega_{0} \times(0, T)} e^{2 s \alpha} t^{-3}(T-t)^{-3}\left|p_{\varepsilon}\right|^{2} d x d t .
\end{aligned}
$$

On the other hand, by the Sobolev imbedding theorem we have for $n=3$ :

$$
\|\nabla z\|_{\frac{3}{1+\delta}} \leq C\|z\|_{H^{\frac{3}{2}-\delta}(\Omega)}, \quad \forall z \in H^{\frac{3}{2}-\delta}(\Omega)
$$

(see $[1,15])$ it follows

$$
\begin{aligned}
\int_{0}^{T} \int_{\Omega}|\nabla w|^{2}\left|p_{\varepsilon} e^{s \alpha}\right|^{2} d x d t & \leq C \int_{0}^{T}\|w(t)\|_{H^{\frac{3}{2}-\delta}(\Omega)}^{2}\left(\int_{\Omega}\left|p_{\varepsilon} e^{s \alpha}\right|^{\frac{6}{1-2 \delta}} d x\right)^{\frac{1-2 \delta}{3}} d t \\
& \leq C M^{2} \int_{0}^{T}\left\|p_{\varepsilon}(t) e^{s \alpha}\right\|_{H^{1+\delta}(\Omega)}^{2} d t .
\end{aligned}
$$

In the same manner as in Section 3 we get that

$$
\begin{aligned}
& C_{1} \rho_{0}(M)^{2} \int_{Q} e^{2 s \alpha}|\nabla w|^{2}\left|p_{\varepsilon}\right|^{2} d x d t \leq \int_{Q} \frac{t(T-t)}{s}\left|\nabla\left(p_{\varepsilon} e^{2 \alpha}\right)\right|^{2} d x d t \\
& +C s^{\frac{2 \delta-1}{1-\delta}}\left[\rho_{0}(M) M\right]^{\frac{2}{1-\delta}} \int_{Q} \frac{s}{t(T-t)}\left|\nabla\left(p_{\varepsilon} e^{s \alpha}\right)\right|^{2} d x d t
\end{aligned}
$$

and after some calculation (analogous to that in Sect. 3) we obtain that

$$
\int_{\Omega}\left|p_{\varepsilon}(x, 0)\right|^{2} d x \leq C e^{C\left[\rho^{2}(M)+\left(\rho_{0}(M) M\right)^{\frac{2}{1-2 \delta}}\right]} \int_{\omega_{0} \times(0, T)}\left|p_{\varepsilon}\right|^{2} \frac{e^{2 s \alpha}}{t^{3}(T-t)^{3}} d x d t
$$

for $s \geq s_{0}+C\left[\rho^{2}(M)+\left(\rho_{0}(M) M\right)^{\frac{2}{1-2 \delta}}\right]$.

The same argument as in the previous section allows us to conclude that

$$
\left\|v_{\varepsilon}\right\|_{H^{2,1}(Q)}^{2} \leq C\left\|y_{0}\right\|_{2}^{2} e^{C\left[\rho^{2}(M)+\left(\rho_{0}(M) M\right)^{\frac{2}{1-2 \delta}}\right]}
$$

(where $v_{\varepsilon}=e^{2 s \alpha} t^{-3}(T-t)^{-3} p_{\varepsilon}$ ) and since $m \in C_{0}^{\infty}(\omega)$ we get that

$$
\left\|m u_{\varepsilon}\right\|_{H^{2,1}(Q)}^{2} \leq C\left\|y_{0}\right\|_{2}^{2} e^{C\left[\rho^{2}(M)+\left(\rho_{0}(M) M\right)^{\frac{2}{1-2 \delta}}\right]} .
$$


On a subsequence we have

$$
\begin{aligned}
& u_{\varepsilon} \longrightarrow u \quad \text { weakly in } H^{2,1}(Q) \\
& y_{\varepsilon} \longrightarrow y \quad \text { weakly in } H^{2,1}(Q)
\end{aligned}
$$

where $(u, y)$ satisfy $(3.2)$ and $y(x, T)=0$, a.e. $x \in \Omega$. Moreover (4.2) implies the estimate in Lemma 4.1 .

Proof of Theorem 2.1 (continued). For each $w \in K$ denote by $\Phi(w) \subset L^{2}(Q)$ the set of all solutions $y^{u} \in H^{2,1}(Q)$ of (3.2) such that $y^{u}(x, T)=0$, a.e. $x \in \Omega$ and

$$
\|m u\|_{H^{2,1}(Q)}^{2} \leq C\left\|y_{0}\right\|_{2}^{2} e^{C\left[\rho^{2}(M)+\left(\rho_{0}(M) M\right)^{\frac{2}{1-2 \delta}}\right]} .
$$

Let $\mathcal{A}$ defined by

We may write (3.2) as

$$
\left\{\begin{array}{l}
D(\mathcal{A})=H_{0}^{1}(\Omega) \cap H^{2}(\Omega) \\
\mathcal{A} y=-\Delta y, \forall y \in D(\mathcal{A})
\end{array}\right.
$$

$$
\left\{\begin{array}{l}
\left(y^{u}\right)^{\prime}+\mathcal{A} y^{u}+a(w) \cdot \nabla y^{u}=m u, \quad t \in(0, T) \\
y^{u}(0)=y_{0}
\end{array}\right.
$$

If we multiply (4.3) by $\mathcal{A}^{1+\gamma} y^{u}$, with $\gamma=\frac{1}{2}-\delta$, we obtain that

$$
\begin{aligned}
& \frac{1}{1+\gamma} \frac{d}{d t}\left(\mathcal{A}^{1+\gamma} y^{u}(t), y^{u}(t)\right)+\left(\mathcal{A} y^{u}(t), \mathcal{A}^{1+\gamma} y^{u}(t)\right) \\
& \leq\|m u(t)\|_{H^{2}(\Omega)} \cdot\left\|\mathcal{A}^{\gamma} y^{u}(t)\right\|_{2}+\left\|\mathcal{A} y^{u}(t)\right\|_{2} \cdot\left\|\mathcal{A}^{\gamma}\left(a(w(t)) \cdot \nabla y^{u}(t)\right)\right\|_{2} \\
& \leq \frac{1}{2}\left(\|m u(t)\|_{H^{2}(\Omega)}^{2}+\left\|\mathcal{A}^{\gamma} y^{u}(t)\right\|_{2}^{2}\right) \\
& +C\left\|\mathcal{A} y^{u}(t)\right\|_{2} \cdot\left\|\nabla\left(a(w(t)) \cdot \nabla y^{u}(t)\right)\right\|_{2}^{2 \gamma} \cdot\left\|a(w(t)) \nabla y^{u}(t)\right\|_{2}^{1-2 \gamma}
\end{aligned}
$$

(we have used the inequality

$$
\left.\left\|\mathcal{A}^{\gamma} z\right\|_{2} \leq C\left\|\mathcal{A}^{\frac{1}{2}} z\right\|_{2}^{2 \gamma} \cdot\|z\|_{2}^{1-2 \gamma}, \quad \forall z \in D\left(\mathcal{A}^{\frac{1}{2}}\right)\right)
$$

Integrating on $(0, t)$ we obtain

$$
\begin{aligned}
\left(\mathcal{A}^{1+\gamma} y^{u}(t), y^{u}(t)\right) & +\int_{0}^{t}\left\|\mathcal{A}^{\frac{1+\gamma}{2}} y^{u}(s)\right\|_{2}^{2} d s \leq C\left(\left\|\mathcal{A}^{\frac{1+\gamma}{2}} y_{0}\right\|_{2}^{2}+\|m u\|_{H^{2,1}(Q)}^{2}+\int_{0}^{t}\left\|\mathcal{A}^{\frac{1}{2}} y^{u}(s)\right\|_{2}^{2} d s\right. \\
& \left.+\rho(M) \int_{0}^{t}\left\|\mathcal{A} y^{u}(s)\right\|_{2}^{1+2 \gamma}\left\|\mathcal{A}^{\frac{1}{2}} y^{u}(s)\right\|_{2}^{1-2 \gamma} d s\right)
\end{aligned}
$$

and consequently

$$
\left\|\mathcal{A}^{\frac{1+\gamma}{2}} y^{u}(t)\right\|_{2}^{2} \leq C\left(\left\|y_{0}\right\|_{H^{1+\gamma}(\Omega)}^{2}+\left\|y_{0}\right\|_{2}^{2} e^{C\left[\rho^{2}(M)+\left(\rho_{0}(M) M\right)^{\frac{2}{1-2 \delta}}\right]}\right) e^{[1+\rho(M)] T} .
$$

Hence

$$
\left\|y^{u}(t)\right\|_{H^{1+\gamma}(\Omega)}^{2} \leq C\left\|y_{0}\right\|_{H^{1+\gamma}(\Omega)}^{2} e^{C\left[\rho^{2}(M)+\left(\rho_{0}(M) M\right)^{\frac{2}{1-2 \delta}}\right]}
$$


a.e. $t \in(0, T)$. As $m u \in H^{2,1}(Q) \subset L^{3}(Q)$ (for $n=3$ ) and $y_{0} \in H^{2}(\Omega) \subset W_{3}^{2-\frac{2}{3}}(\Omega)$ we may conclude that

$$
\left\|y^{u}\right\|_{L^{\infty}(Q)}^{2} \leq C\left\|y^{u}\right\|_{W_{3}^{2,1}(Q)}^{2}
$$

(we recall that $W_{3}^{2,1}(Q) \subset L^{\infty}(Q)$ for $n=3$; see [13] and [15])

$$
\leq C\left(\left\|y_{0}\right\|_{H^{2}(\Omega)}^{2}+\|m u\|_{H^{2,1}(Q)}^{2}+\rho^{2}(M)\left\|\nabla y^{u}\right\|_{L^{2}(Q)}^{2}\right) \leq C\left\|y_{0}\right\|_{H^{2}(\Omega)}^{2} e^{C\left[\rho^{2}(M)+\left(\rho_{0}(M) M\right)^{\frac{2}{1-2 \delta}}\right]} .
$$

Thus it follows by (4.4) and (4.5) that if

$$
\left\|y_{0}\right\|_{H^{2}(\Omega)} \leq C M e^{-C\left[\rho^{2}(M)+\left(\rho_{0}(M) M\right)^{\frac{2}{1-2 \delta}}\right]}
$$

then $\Phi(K) \subset K$.

In the same manner as in Section 3 it follows that $\Phi(w)$ is a closed and convex subset of $L^{2}(Q)$, for any $w \in K$ and $\Phi$ is upper semicontinuous in $L^{2}(Q) \times L^{2}(Q)$.

Applying the Kakutani fixed point theorem (see [2], p. 7 and [6], p. 310) in the space $L^{2}(Q)$ to the mapping $\Phi$, we infer that there is at least one $w \in K$ such that $w \in \Phi(w)$. This implies that there is at least one pair $(u, y)$ satisfying the conditions of Theorem 2.1.

Since $(2.2)^{\prime}$ is satisfied we may infer that if

$$
\left\|y_{0}\right\|_{H^{2}(\Omega)}<C \sup \left\{r e^{-C\left[\rho^{2}(r)+\left(\rho_{0}(r) r\right)^{\frac{2}{1-2 \delta}}\right]} ; r>0\right\}=+\infty,
$$

then there is at least one pair $(u, y)$ satisfying the conditions of Theorem 2.1 and this completes the proof.

Remark 4.2. The analysis of the proof of Theorem 2.1 shows that in the above argument the condition $n \in\{1,2,3\}$ cannot be dispensed with. However, one might speculate that this could be true by using instead of (3.3) the Carleman estimate proved in [12] (Th. 2.1), i.e.,

$$
\begin{gathered}
s^{-1} \int_{Q} t(T-t) e^{2 s \alpha}\left|\nabla p_{\varepsilon}\right|^{2} d x d t+s \int_{Q}(t(T-t))^{-1} e^{2 s \alpha}\left|p_{\varepsilon}\right|^{2} d x d t \\
\leq C\left(\|w\|_{L^{\infty}(Q)}^{2} \int_{Q} e^{2 s \alpha}\left|p_{\varepsilon}\right|^{2} d x d t+s \int_{\omega \times(0, T)} e^{2 s \alpha}(t(T-t))^{-1}\left|p_{\varepsilon}\right|^{2} d x d t\right),
\end{gathered}
$$

for $s \geq s_{0}$.

For $s \geq s_{0}+\rho^{2}(M)$ we obtain

$$
s^{-1} \int_{Q} t(T-t) e^{2 s \alpha}\left|\nabla p_{\varepsilon}\right|^{2} d x d t+s \int_{Q}(t(T-t))^{-1} e^{2 s \alpha}\left|p_{\varepsilon}\right|^{2} d x d t \leq C s \int_{\omega \times(0, T)} e^{2 s \alpha}(t(T-t))^{-1}\left|p_{\varepsilon}\right|^{2} d x d t
$$

and therefore (compare with (3.16))

$$
\int_{\Omega}\left|p_{\varepsilon}(x, 0)\right|^{2} d x \leq C \rho^{2}(M) e^{C \rho^{2}(M)}\left\|y_{0}\right\|_{2}^{2} \int_{\omega \times(0, T)} e^{2 s \alpha} s(t(T-t))^{-1}\left|p_{\varepsilon}\right|^{2} d x d t .
$$

Thus if one replace problem $\left(P_{1 \varepsilon}\right)$ by

$$
\text { Minimize } \int_{Q} e^{-2 s \alpha} t(T-t) u^{2} d x d t+\frac{1}{\varepsilon} \int_{\Omega} y^{2}(x, T) d x
$$


subject to (3.2) and if we denote by $\left(u_{\varepsilon}, y_{\varepsilon}\right)$ an optimal pair, then we get

$$
\int_{\omega \times(0, T)} e^{-2 s \alpha} t(T-t) u_{\varepsilon}^{2} d x d t+\frac{1}{\varepsilon} \int_{\Omega} y_{\varepsilon}^{2}(x, T) d x \leq C \rho^{2}(M) e^{C \rho^{2}(M)}\left\|y_{0}\right\|_{2}^{2}
$$

and $u_{\varepsilon}=m p_{\varepsilon} e^{2 s \alpha} t^{-1}(T-t)^{-1}$.

If $n=1$, this is enough to conclude that (3.21) holds, but we can not obtain the same inequality for $n \geq 2$. The reason is that (4.6) and (4.7) are not sufficiently sharp to imply a uniform bound for $\left\{u_{\varepsilon}\right\}$ in $L^{l}(Q)$ for $l$ sufficiently large. In fact, having in mind the proof of (3.18) we need besides (4.6) an uniform estimate for $\left(p_{\varepsilon}\right)_{t}$ and $\Delta p_{\varepsilon}$, which could not be obtained by (4.6).

Remark 4.3. An analysis of the previous proof reveals that condition $1 \leq n \leq 3$ cannot be dispensed with. In fact in the proof of Lemma 4.1, for $n \geq 4$, inequality (4.1) remains true for $\delta>\frac{1}{2}$ only. On the other hand, $\int_{0}^{T}\left\|p_{\varepsilon}(t) e^{s \alpha}\right\|_{H^{1+\delta}}^{2}(\Omega) d t$ cannot be dominated by the left side term in the Carleman inequality and so the subsequent estimates do not hold.

\section{Proof of Theorem 2.3}

We shall prove first the following lemma

Lemma 5.1. Let $0<\eta<T$ be arbitrary but fixed and $y_{0} \in L^{2}(\Omega)$. Then the system

$$
\begin{cases}y_{t}-\Delta y+a(y) \cdot \nabla y=0, & (x, t) \in \Omega \times(0, \eta)=Q_{\eta} \\ y=0, & (x, t) \in \partial \Omega \times(0, \eta)=\Sigma_{\eta} \\ y(x, 0)=y_{0}(x), & x \in \Omega,\end{cases}
$$

has at least one solution

$$
y \in C\left([0, \eta] ; L^{2}(\Omega)\right) \cap L^{2}\left(0, \eta ; H_{0}^{1}(\Omega)\right) \cap L^{2}\left(\varepsilon, \eta ; H^{2}(\Omega)\right)
$$

(for any $0<\varepsilon<\eta)$, $a(y) \cdot \nabla y \in L^{1}\left(Q_{\eta}\right)$ and $a(y) \cdot \nabla y \in L^{2}(\Omega \times(\varepsilon, \eta))$.

Proof. Let $\left\{y_{0 N}\right\}_{N \in \mathbf{N}^{*}} \subset H_{0}^{1}(\Omega) \cap H^{2}(\Omega)$ be such that $y_{0 N} \rightarrow y_{0}$ in $L^{2}(\Omega)$, as $N \rightarrow+\infty$ and let

$$
y_{N} \in C\left([0, \eta] ; L^{2}(\Omega)\right) \cap H^{2,1}(\Omega \times(0, \eta))
$$

be the unique solution to (5.1), corresponding to $y_{0}:=y_{0 N}$.

Note the a priori estimates

$$
\left\|y_{N}\right\|_{C\left([0, \eta] ; L^{2}(\Omega)\right)}+\left\|y_{N}\right\|_{L^{2}\left(0, \eta ; H_{0}^{1}(\Omega)\right)}+\left\|a\left(y_{N}\right) \cdot \nabla y_{N}\right\|_{L^{1}(\Omega \times(0, \eta))} \leq C,
$$

for all $N \in \mathbf{N}^{*}$. This implies by standard arguments (see [5]) that

$$
\begin{array}{ll}
y_{N} \rightarrow y & \text { strongly in } L^{q}(\Omega \times(0, \eta)) \\
\nabla y_{N} \rightarrow \nabla y & \text { weakly in } L^{2}(\Omega \times(0, \eta))
\end{array}
$$

(for any $\left.1<q<\frac{n+2}{n}\right)$. Since $\left\{y_{N}\right\}$ is bounded in $C\left([0, \eta] ; L^{2}(\Omega)\right)$ and in $L^{2}\left(0, \eta ; L^{6}(\Omega)\right)\left(H_{0}^{1}(\Omega) \subset L^{6}(\Omega)\right)$, then it follows that $\left\{y_{N}\right\}$ is bounded in $L^{3}(\Omega \times(0, \eta))$. Indeed,

$$
\int_{0}^{\eta} \int_{\Omega}\left|y_{N}(x, t)\right|^{3} d x d t \leq \int_{0}^{\eta}\left\|y_{N}(t)\right\|_{6}^{3 / 2} \cdot\left\|y_{N}(t)\right\|_{2}^{3 / 2} d t \leq\left\|y_{N}\right\|_{L^{2}\left(0, \eta ; L^{6}(\Omega)\right)}^{\frac{3}{2}}\left\|y_{N}\right\|_{L^{6}\left(0, \eta ; L^{2}(\Omega)\right)}^{\frac{3}{2}} .
$$


Since $\left\{y_{N}\right\}$ is bounded in $L^{3}\left(Q_{\eta}\right)$ and $y_{N} \rightarrow y$ in $L^{q}(\Omega \times(0, \eta))$, then we conclude that

$$
y_{N} \rightarrow y \quad \text { strongly in } L^{2}(\Omega \times(0, \eta)) .
$$

It is now obvious that

$$
a\left(y_{N}\right) \cdot \nabla y_{N} \rightarrow a(y) \cdot \nabla y \quad \text { weakly in } L^{1}(\Omega \times(0, \eta))
$$

and so we may pass to the limit in (5.1) (with $y_{0}:=y_{0 N}$ ).

By $L^{k}$ regularity of parabolic equations it also follows that

$$
y \in C\left([0, \eta] ; L^{2}(\Omega)\right) \cap L^{2}\left(0, \eta ; H_{0}^{1}(\Omega)\right) \cap L^{2}\left(\varepsilon, \eta ; H^{2}(\Omega)\right),
$$

$a(y) \cdot \nabla y \in L^{1}\left(Q_{\eta}\right) \cap L^{2}(\Omega \times(\varepsilon, \eta)), \forall \varepsilon \in(0, \eta)$ and $y$ is a solution to (5.1).

Proof of Theorem 2.3 (continued). Let $y_{0} \in L^{2}(\Omega)$. Then by Lemma 5.1 there is a solution $\bar{y}$ to (5.1) on $\Omega \times(0, \nu)(\nu \in(0, \eta))$ such that

$$
\bar{y} \in C\left([0, \nu] ; L^{2}(\Omega)\right) \cap L^{2}\left(0, \nu ; H_{0}^{1}(\Omega)\right) \cap L^{2}\left(\varepsilon, \nu ; H^{2}(\Omega)\right),
$$

for all $\varepsilon \in(0, \nu)$ and $y_{1}=\bar{y}(\nu) \in H_{0}^{1}(\Omega) \cap H^{2}(\Omega)$.

On the other hand, by Theorem 2.1 there is $(\tilde{y}, \tilde{u}) \in H^{2,1}(\Omega \times(\nu, T)) \times L^{2}(\Omega \times(\nu, T))$, which satisfy (1.1) on $\Omega \times(\nu, T), \tilde{y}(x, \nu)=y_{1}(x)$ and $\tilde{y}(x, T)=0$ a.e. $x \in \Omega$.

We set

$$
\begin{aligned}
& u^{*}(x, t)= \begin{cases}0, & (x, t) \in \Omega \times(0, \nu) \\
\tilde{u}(x, t), & (x, t) \in \Omega \times(\nu, T),\end{cases} \\
& y^{*}(x, t)= \begin{cases}y(x, t), & (x, t) \in \Omega \times(0, \nu) \\
\tilde{y}(x, t), & (x, t) \in \Omega \times(\nu, T) .\end{cases}
\end{aligned}
$$

Clearly $\left(y^{*}, u^{*}\right)$ satisfy $(1.1)$ on $\Omega \times(0, T)$ and satisfy conditions of Theorem 2.3.

This completes the proof.

The authors express their thanks to Professor E. Zuazua for several suggestions and remarks which improved the presentation of this paper.

\section{REFERENCES}

[1] R.A. Adams, Sobolev Spaces. Academic Press, New York (1975).

[2] V. Barbu, Analysis and Control of Nonlinear Infinite Dimensional Systems. Academic Press, Boston (1993).

[3] V. Barbu, Exact controllability of the superlinear heat equation. Appl. Math. Optim., to appear.

[4] V. Barbu, T. Precupanu, Convexity and Optimization in Banach Spaces. D. Reidel Publ. Company, Dordrecht (1986).

[5] H. Brézis and A. Friedman, Nonlinear parabolic equations involving measures as initial conditions. J. Math. Pures Appl. 62 (1983) 73-97.

[6] K. Deimling, Nonlinear Functional Analysis. Springer-Verlag, Berlin (1985).

[7] C. Fabre, J.P. Puel and E. Zuazua, Approximate controllability of the semilinear heat equation, Proceedings Royal Soc. Edinburgh 125 A (1995) 31-61.

[8] E. Fernández-Cara, Null controllability of the semilinear heat equation. ESAIM Control. Optim. Calc. Var. 2 (1997) 87-107.

[9] E. Fernández-Cara and E. Zuazua, The cost of approximate controllability for heat equations: The linear case. Adv. Diff. Equations, to appear.

[10] E. Fernández-Cara and E. Zuazua, Null and approximate controllability for weakly blowing up semilinear heat equations. Ann. Inst. H. Poincaré Anal. Non Linéaire, to appear.

[11] A.V. Fursikov and O.Yu. Imanuvilov, Controllability of Evolution Equations. RIM Seoul National University, Korea, Lecture Notes Ser. 34 (1996). 
[12] O.Yu. Imanuvilov and M. Yamamoto, On Carleman inequalities for parabolic equations in Sobolev spaces of negative order and exact controllability for semilinear parabolic equations, preprint \#98 - 46. University of Tokyo, Grade School of Mathematics, Komobo, Tokyo, Japan (1998).

[13] O.A. Ladyzenskaya, V.A. Solonnikov and N.N. Uraltzeva, Linear and Quasilinear Equations of Paraboic Type. Nauka, Moskow (1967).

[14] G. Lebeau and L. Robbiano, Contrôle exact de l'équation de la chaleur. Comm. Partial Differential Equations 30 (1995) $335-357$.

[15] J.L. Lions, Contrôle des systèmes distribués singuliers, MMI 13. Gauthier-Villars (1983).

[16] J.L. Lions and E. Magenes, Problèmes aux limites non homogènes et applications, Vol. 1. Dunod, Paris (1968).

[17] E. Zuazua, Approximate controllability of the semilinear heat equation: boundary control, in Computational Sciences for the 21st Century, M.O. Bristeau et al., Eds. John Wiley \& Sons (1997) 738-747.

[18] E. Zuazua, Approximate controllability for semilinear heat equations with globally Lipschitz nonlinearities. Control Cybernet., to appear. 